\title{
Unusual presentation of atrial Myxoma in a young Malawian male: Case report and review of literature
}

\author{
Geoffrey Elihu Manda ${ }^{1}$, Mwai Mtekateka ${ }^{1,2,}$ Sharon Kunkanga ${ }^{1}$, Noel Kayange ${ }^{1,2,3}$
}

1. Queen Elizabeth Central Hospital, Blantyre, Malawi.

2. University of Malawi, College of Medicine, Blantyre Malawi.

3. John Hopkins Research Project, Blantyre, Malawi.

Correspondence: Geoffrey Elihu Manda; gmanda@medcol.mw

\section{Introduction}

We report a 30-year-old male who presented with signs and symptoms of right heart failure who was later diagnosed with right-sided atrial myxoma. This patient, unfortunately, died while waiting for cardiac surgery outside Malawi and postmortem was not done to ascertain the cause of death.

\section{Clinical case}

A 30-year-old male was admitted to Queen Elizabeth Central Hospital, Malawi with a two-week history of sudden onset, worsening shortness of breath on exertion and at rest with associated fatigue and dizziness but no syncope. This was followed by swelling of the legs to the level of the thighs but was without symptoms of chest pain, orthopnea, paroxysmal nocturnal dyspnea, or cough. He had a 10-year history of drinking locally brewed spirits (kachasu) and a 12-year history of smoking unfiltered tobacco (chingambwe) which could not be quantified objectively. There was no other relevant history and systemic review was unremarkable. On physical examination, he was afebrile, had a normal BMI of $23 \mathrm{Kg} /$ M2, was tachypneic, had a normal oxygen saturation of $99 \%$ on room air and was normotensive with normal and regular heart rate. He had mucocutaneous pallor and bilateral pitting oedema extending from the feet to the thighs with no sacral oedema, cyanosis, or lymphadenopathy. He had a raised Jugular Venous Pressure of $12 \mathrm{~cm} \mathrm{H} 0$, had a hyperactive precordium with a non-displaced apex heartbeat and a right ventricular heave. On auscultation, he had a grade 3, nonradiating ejection systolic murmur heard best at the left lower sternal edge. Abdominal examination revealed free fluid suggestive of ascites but no clinical evidence of hepatic or splenic enlargement. The rest of the physical examination was normal. Full blood count showed normocytic anaemia of $10 \mathrm{~g} / \mathrm{dl}$ and other parameters were normal. The following tests were either normal or negative: liver and renal function tests, serum electrolytes, HIV, hepatitis B and C and syphilis serology.

A Trans-thoracic echocardiogram (T'TE) showed a vascular right atrial mass attached to the fossa ovalis region in the inter-atrial septum measuring $6 \mathrm{~cm}$ by $4 \mathrm{~cm}$ with cystic core prolapsing in tandem with heart contraction through the tricuspid valve at least a third into right ventricle in diastole causing severe tricuspid stenosis. There was a pericardial effusion of $1.1 \mathrm{~cm}$ causing no cardiac tamponade. Aortic and mitral valve morphology and left ventricular ejection fraction (65\%-75\%) were normal (Figure 1). Chest radiography, abdominal ultrasound scan and electrocardiography were normal.

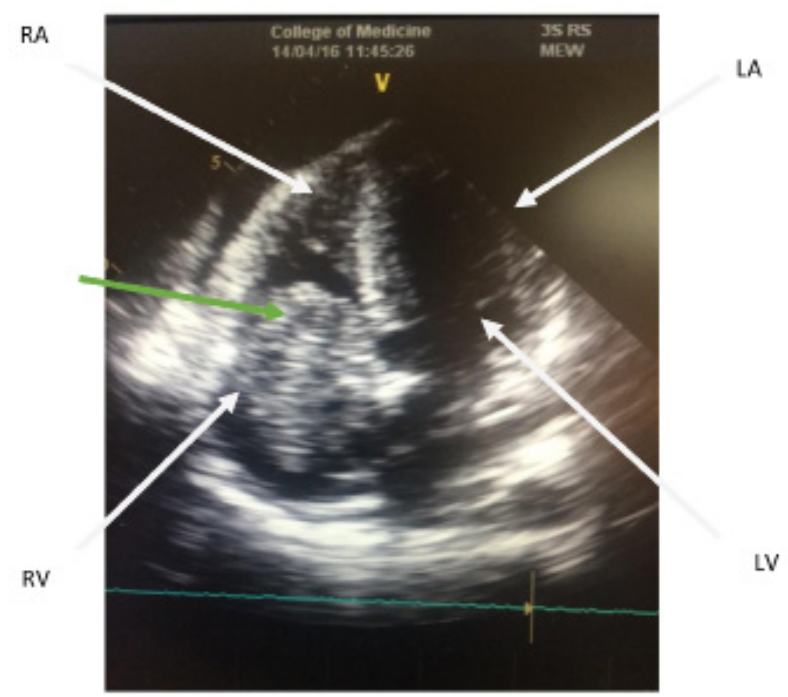

Figure 1. Four Chamber view showing right atrial myxoma (green arrow). $($ Right atrium $=\mathrm{RA}$, right ventricle $=\mathrm{RV}$, left atrium $=\mathrm{LA}$ and left ventricle $=\mathrm{LV}$ )

Diagnosis and management

Based on the clinical findings and echocardiography a diagnosis of right heart failure secondary to right atrial myxoma was made. Fluid overload was managed with intravenous furosemide $(40 \mathrm{mg}$ ) twice daily with rapid clinical improvement of symptoms within three days. Unfractionated Subcutaneous Heparin (7500 Units) twice daily was also added for thromboprophylaxis. While waiting for cardiac surgery outside Malawi the patient's condition deteriorated and he later died.

\section{Discussion}

Primary intra-cardiac tumours occur infrequently with a prevalence of $0.02 \%$ of the general population in autopsy studies ${ }^{1}$. In comparison, secondary cardiac tumours are estimated to be 20 to 30 times more common however, their exact incidence is unknown ${ }^{2,3}$. Benign primary cardiac tumours are more common $(75 \%)^{3}$ with approximately half being atrial myxomas and left-sided being the commonest $(75-80 \%)^{4}$. They occur commonly in females $(65 \%-70 \%)$ and can be seen in patients of all age groups but the peak incidence is in the fourth and sixth decade of $l i f e^{5-7}$. They can be multiple $(5 \%)$ of which half are bi-atrial ${ }^{6,8}$. Reports of atrial myxoma from Africa are scarce and the exact 
epidemiology in Africa and Malawi is unknown.

Familial myxomas are rare $(7 \%)$ and present early in life mostly as Carney syndrome because of mutation of the PRKAR1 $\alpha$ gene located on the long arm of chromosome 17 (17q22-24 region) and transmitted in an autosomal dominant fashion? Myxomas are thought of as neoplasms of endocardial origin arising from pluripotent mesenchymal cells associated with embryonic residues in the sub-endocardium and but they generally appear to grow rather quickly ${ }^{10}$. As was with our case, most myxomas are seen at a narrow attachment point at the fossa ovalis of the inter-atrial septum-with a tendency to prolapse into the Mitral Apparatus ${ }^{10,11}$. Though limited in the field of view, a 2-dimensional TTE is a first-line modality to detect cardiac tumours. More superior but unavailable in our setting is the trans-oesophageal echocardiography (TOE) approach which can provide unimpeded visualization of the atria, atrial septum, and portions of the left and right ventricles and can detect small vegetation and tumours 12. Chest radiography though normal in this patient may reveal right or left atrial enlargement or calcifications. The clinical features of myxomas are determined by their location, size, and mobility. Occasionally (20\%) there are no symptoms, particularly with small tumours but large ones present with one or more of the triads of embolism, intracardiac obstruction, and constitutional symptoms ${ }^{4}$. In Leftsided atrial myxomas patients may present with signs and symptoms that mimic mitral stenosis, leading to dyspnea or orthopnea. If the tumour is large enough, temporary complete obstruction of the orifice of the mitral or tricuspid valve may occur resulting in syncope $(20 \%)$ or sudden death. Systemic embolism occurs in $30-40 \%$ of patients with large and friable myxomas ${ }^{11}$ and thromboprophylaxis was instituted in our patient in accordance. Our patient also presented with some constitutional disturbances, such as fatigue, dizziness, and laboratory abnormalities, such as anaemia suggesting a possible infection or the primary malignant disease. Sometimes myxomas can get infected increasing the risk of systemic embolization. Our patient died while waiting for surgery outside Malawi due to a lack of cardiothoracic surgery facilities in Malawi although the prognosis is much better after surgical resection ${ }^{10}$.

\section{Conclusion}

Atrial myxomas are very rare but should be considered in any patient presenting with heart failure. The diagnosis of atrial myxoma can be elusive, especially when the symptoms are non-specific. Although easily treated in the western world, surgical intervention is not widely available in Malawi and most regions of Africa ${ }^{13}$. We recommend an echocardiogram in the workup of heart failure of unknown aetiology to facilitate earlier identification of intra-cardiac growths for timely intervention. The unfortunate outcome of this case (patient death) highlights that young, otherwise healthy, Malawian patients are dying from easily treatable cardiac diseases because of the lack of cardiac surgery in Malawi.

\section{Acknowledgement}

We would like to acknowledge all colleagues for their helpful contribution to the care of this patient.

\section{References}

1. Amano J, Nakayama J, Yoshimura Y, Ikeda U. Clinical classification of cardiovascular tumours and tumour-like lesions, and its incidences. Gen Thorac Cardiovasc Surg. 2013;61(8):435-47. doi: 10.1007/ s11748-013-0214-8

2. Bussani R, De-Giorgio F, Abbate A, Silvestri F. Cardiac metastases. J Clin Pathol. 2007;60(1):27-34. doi: 10.1136/jcp.2005.035105

3. Leja MJ, Shah DJ, Reardon MJ. Primary Cardiac Tumors. Tex Heart Inst J. 2011;38(3):261-2. PMCID: PMC3113129

4. Isobe S, Murohara T. Editorial: Cardiac tumours: Histopathological aspects and assessments with cardiac noninvasive imaging. J Cardiol Cases. 2015;12(2):37-8. DOI: https://doi.org/10.1016/j. jecase. 2015.04 .008

5. Pinede L, Duhaut P, Loire R. Clinical presentation of left atrial cardiac myxoma. A series of 112 consecutive cases. Medicine (Baltimore). 2001;80(3):159-72. PMID: 11388092

6. Azari A, Moravvej Z, Chamanian S, Bigdelu L. An Unusual Biatrial Cardiac Myxoma in a Young Patient. Korean J Thorac Cardiovasc Surg. 2015;48(1):67-9. doi: 10.5090/kjtcs.2015.48.1.67

7. Kumar R, Jaiswal A, Mehrotra R. Large left atrial myxoma causing significant mitral regurgitation. J Indian Acad Echocardiogr Cardiovasc Imaging. 2017;1(1):55. DOI: 10.4103/jiae.JIAE_18_17

8. Vijan V, Vupputuri A, Chandrasekharan Nair R. An Unusual Case of Biatrial Myxoma in a Young Female. Case Rep Cardio 2016;2016:3545480. doi: http://dx.doi.org/10.1155/2016/3545480

9. Forlino A, Vetro A, Garavelli L, Ciccone R, London E, Stratakis CA, et al. PRKACB and Carney Complex. N Engl J Med. 2014;370(11):10657. DOI: $10.1056 / \mathrm{NEJMc} 1309730$

10. Reynen K. Cardiac Myxomas. N Engl J Med. 1995;333(24):16107. DOI: 10.1056/NEJM199512143332407

11. Wu PW, Chen S-J, Lin W-C, Chang C-B, Chuang W-Y, Hsu M-Y, et al. Imaging of Cardiac and Pericardial Masses. Cardiovasc Imaging Asia. 2017;1(1):23-37.doi: https://doi.org/10.22468/cvia.2016.00059

12. Celeste F, Muratori M, Mapelli M, Pepi M. The Evolving Role and Use of Echocardiography in the Evaluation of Cardiac Source of Embolism. J Cardiovasc Echography. 2017;27(2):33-44. doi: 10.4103/ jcecho.jcecho_1_17.

13. Peter McKavanagh, Karen Booth, Bharathi Varadarajan, and O C Nzewi. Advanced atrial myxoma in rural African setting. BMJ Case Rep. 2014; 2014: bcr2014206863. doi: 10.1136/bcr-2014-206863 